\title{
Der physische Raum im Kontext der Digitalisierung. Perspektiven für Lehr- und Lernraumkonstellationen an Hochschulen
}

\section{Richard Stang, Anke Petschenka, Christine Gläser und Alexandra Becker}

\section{Zusammenfassung}

Im Zuge der Coronapandemie hat sich gezeigt, welche Relevanz Strategien für das Onlinelehren und -lernen für Hochschulen haben. Doch greift es zu kurz, nur den digitalen Raum in strategische Überlegungen bei der Hochschulentwicklung einzubeziehen. Auch der physische Raum wird in Zukunft von zentraler Bedeutung für die Gestaltung der Rahmenbedingungen für Lernende sein. Es gilt, hybride Lehr- und Lernraumkonstellationen zu entwickeln. Dabei wird die Einbeziehung der Studierendenperspektive besonders relevant. Der Beitrag entfaltet die verschiedenen Dimensionen einer entsprechenden Strategieentwicklung an Hochschulen im 21. Jahrhundert. Auf der Basis der Forschungsergebnisse des Projektes „Lernwelt Hochschule (LeHo)“ werden

R. Stang $(\varangle) \cdot$ A. Becker

Hochschule der Medien, Stuttgart, Deutschland

E-Mail: stang@hdm-stuttgart.de

A. Becker

E-Mail: beckera@hdm-stuttgart.de

A. Petschenka

Universität Duisburg-Essen, Essen, Deutschland

E-Mail: anke.petschenka@uni-due.de

C. Gläser

HAW Hamburg, Hamburg, Deutschland

E-Mail: Christine.Glaeser@haw-hamburg.de 
ausgewählte Aspekte diskutiert, die für Hochschulen von besonderer Relevanz sind, wenn sie im Modus der Digitalisierung Zukunft gestalten.

\section{Schlüsselwörter}

Physischer Lehr- und Lernraum • Virtueller Lehr- und Lernraum • Innovative Lernkonzepte • Hybride Lehr- und Lernraumkonzepte • Student Experience • Lernraumtheorie

\section{Gestaltung von Raumszenarien als Herausforderung}

Gesellschaftliche Veränderungsprozesse fordern in besonderer Weise Bildungseinrichtungen und damit auch Hochschulen heraus. Spätestens mit den Folgen der Coronapandemie rückt das Thema Onlinelehren und Onlinelernen und damit die Digitalisierung stärker in den Fokus von Lehrenden und Studierenden. Der digitale Raum ist jedoch nur ein Bereich, der für die Zukunft von Bildung von Relevanz ist. Die physischen Lehr-Lernräume spielen gleichermaßen eine große Rolle, da räumliche Faktoren Lernen immer beeinflussen. Lernende lernen immer im Kontext der Verortung ihres analogen Körpers. So stellt der physische Raum einen zentralen Erlebnis- und Erfahrungskontext beim Lernen dar, auch wenn mit oder an digitalen Medien gelernt wird (Stang 2017). Doch wie genau gestaltet sich die Situation an den deutschen Hochschulen bezogen auf die Struktur der Lehr-Lernräume?

Das Projekt „Lernwelt Hochschule“1, das von der Hochschule der Medien Stuttgart (HdM) in Zusammenarbeit mit der Hochschule für Angewandte Wissenschaften Hamburg, der Otto-Friedrich-Universität Bamberg, der Heinrich-HeineUniversität Düsseldorf und der Deutschen Initiative für Netzwerkinformation e. V. (DINI) realisiert und von der Dieter Schwarz Stiftung finanziert wurde, hat über drei Jahre unter anderem die Situation bezogen auf die Lehr-Lernräume an deutschen Hochschulen untersucht (Becker und Stang 2020a; Stang und Becker 2020). Im Fokus stand dabei die Frage, inwieweit der Shift from Teaching to Learning in Deutschland zu mehr Studierendenorientierung auch in Bezug auf die Gestaltung von Lehr- und Lernräumen geführt hat. Methodisch wurden sowohl Umfragen als auch qualitative Expert*inneninterviews im In- und Ausland und ethnografische

\footnotetext{
${ }^{1}$ Siehe dazu: http: www.zukunftlernwelthochschule.de. Zugegriffen: 19.10.2020.
} 
Fallstudien an unterschiedlichen deutschen Hochschulen durchgeführt (Aschinger et al. 2020).

Auf der Basis der Forschungsergebnisse lässt sich feststellen, dass einige Aktivitäten angestoßen wurden, aber noch keine hinreichende Umorientierung und Dynamik zu erkennen ist. Eine Neuausrichtung der Lehre im Hinblick auf mehr Studierendenorientierung ist nur punktuell festzustellen. Dies spiegelt sich auch in der Gestaltung digitaler Strukturen und physischer Lehr- und Lernräume wider. Nur langsam entstehen Raumszenarien, die den Anforderungen von projektorientiertem und forschendem Lernen Rechnung tragen. Es könnten jedoch dynamischere Prozesse flächendeckend vonseiten der Hochschulleitungen angeregt werden, so wie es sich an einigen Hochschulen bereits abzeichnet.

In diesem Beitrag werden einige Forschungsergebnisse vorgestellt und die Herausforderungen im Kontext des raumtheoretischen Diskurses eingeordnet. Dabei werden auch Raumanforderungen in Bezug auf innovative Lehr- und Lernformate und die Betreuung in den Blick gerückt. Wie Studierende stärker in die Entwicklung und Planung von Lehr-Lernräumen an Hochschulen eingebunden werden können, wird unter der Perspektive von Student Experience als Grundlage von Planungsprozessen beleuchtet.

\section{Lernraumtheoretischer Zugang und Lernraumkonzepte}

Eine der zentralen Fragen, die sich im Kontext der Gestaltung von Lehr- und Lernraumkonzepten stellt, lautet: Auf welcher pädagogischen Basis wird die Entwicklung von Lehr- und Lernräumen an Hochschulen gestaltet? Die Raumstrukturen der Lernwelt Hochschule sind vielfältig. Sie reichen von Hörsälen bis hin zu flexiblen Raumarrangements, in denen Lernen selbst organisiert stattfinden kann. Im pädagogischen Kontext ist der Diskurs über Lehr- und Lernräume von besonderer Relevanz.

In der Erziehungswissenschaft wurde die Raumthematik erst im Anschluss an die Diskussion des sogenannten Spatial Turn in den Kultur- und Sozialwissenschaften in den 1990er-Jahren intensiver in den Blick genommen (Kraus et al. 2015, S. 11). Unter historischer Perspektive betrachtet ist die Frage der pädagogischen Gestaltung des Raums für alle Bildungsbereiche schon immer von Relevanz (Jelich und Kemnitz 2003). Ein wichtiger Perspektivenwechsel erfolgte, als die Aneignungspraxis in Bezug auf Räume verstärkt in den Blick genommen wurde. Für Nugel entsteht ,ein pädagogischer Raum erst durch die Aneignungspraxis der einzelnen Lernenden“ (Nugel 2015, S. 62). Er schließt damit an die Überlegungen 
von Löw an, für die sich Raum erst, ,in der Wechselwirkung zwischen Handeln und Strukturen“ (Löw 2012, S. 191) konstituiert. Der Lehr- und Lernraum wird zum sozialen Raum, der nicht nur durch die Gestaltung, Möblierung und Raumorganisation (vor)definiert ist, sondern auch durch das Agieren der Lernenden und Lehrenden. Die Berücksichtigung dieser Wechselwirkung ist bei der Gestaltung von Lehr- und Lernsettings im Bildungsbereich - auch im Hochschulbereich - von besonderer Bedeutung.

Bereits seit Anfang der 2000er-Jahre wurde die Diskussion über die Zukunft von Hochschulen in Großbritannien intensiv in Bezug auf die Entwicklung von Raumkonzepten geführt (JISC 2006; UCISA 2016). Vor welchen Herausforderungen die Hochschulen stehen, wurde im „UK Higher Education Learning Space Toolkit" dargestellt:

\begin{abstract}
„Universities have recognised the need for investment in both formal and informal learning spaces to support the student experience and this includes the requirement to balance innovative and collaborative spaces with traditional tiered lecture theatres, which are increasingly being adapted to new learning practices and continue to play a useful role in the teaching of large cohorts. We are moving away from desk and chair workspaces to providing a range of types of furnishing and deploying a variety of technologies. We are also seeing a shift in where these facilities are located with the development of more learning hubs and satellites“ (UCISA 2016, S. 5).
\end{abstract}

Doch an welchem Bild von Lernen orientiert sich die Gestaltung von LehrLernräumen? Hörsäle, Seminarräume, Labore und Bibliotheken gehören zur klassischen räumlichen Strukturierung von Hochschulen. Dabei orientieren sich die meisten Lehr- und Lernräume an einem sehr traditionellen Verständnis von Lernen: Die Lehrenden vermitteln Wissen, das die Lernenden lernen sollen. Die Raumgestaltung ist hier meistens auf die Lehrenden ausgerichtet. Rücken allerdings die Lernenden in den Mittelpunkt und die Lehrenden begleiten den Lernprozess und beraten die Lernenden, werden flexible Raumarrangements und offene Lernlandschaften notwendig, mit denen auf verschiedenste Lernsituationen reagiert werden kann und die an vielfältigste Aufgabenstellungen angepasst werden können (Stang 2016, S. 24 f.).

Will man an Hochschulen allen Lehr- und Lernmodi Rechnung tragen, bedeutet dies, dass unterschiedliche Raumtypen beziehungsweise Raumfunktionen vorgehalten werden sollten. Brandt und Bachmann sehen den Bedarf an folgenden Raumtypen:

- „Lehrräume für die Durchführung und Organisation der Lehrveranstaltungen,

- Lernräume für selbstgesteuertes Lernen, allein oder in Gruppen, 
- Zwischenräume, in denen sich Studierende erholen, mit anderen austauschen und verpflegen können,

- Prüfungsräume für die Durchführung der zahlreicher gewordenen und oft zeitgleichen Prüfungen,

- Spielräume, um innovative Lehr- und Lernformen zu entwickeln und umzusetzen“ (Brandt und Bachmann 2014, S. 16, Hervorhebungen im Original).

Doch liefert eine solche Typisierung erneut eine Strukturierung, die einer ganzheitlichen Perspektive, die den Student-Life-Cycle einbezieht, nicht gerecht wird. Auch wird der digitale Raum hier nicht aufgeführt, dessen Bedeutung in den letzten Jahren im Hochschulkontext deutlich zugenommen hat. Hierin liegen auch einige Grundprobleme des Raumdiskurses in Bildungseinrichtungen - also auch an Hochschulen -, in dem der digitale und der physische Raum oft in Abgrenzung zueinander gesehen werden. Doch kann Lernen im Modus des Digitalen nicht ohne die physische Verortung stattfinden und das Lernen im Modus des Physischen/Analogen findet zunehmend in Vernetzung zum Digitalen statt. Hier bedarf es für die Zukunft Konzepte, die Hochschule als hybriden Lehr- und Lernraum in den Blick nehmen. Dies bedeutet, dass Integration und gegenseitige Bezüge von physischen und digitalen Lernraumelementen für das Lehren und Lernen sinnvoll hergestellt werden.

Wichtige Grundlagen werden dabei differenzierte pädagogische beziehungsweise didaktische Konzeptionen sein, die sich auch am Bedarf von projektorientiertem und problembasiertem Lernen orientieren, da gerade hier Kompetenzentwicklung auf besondere Weise gefördert werden kann (Stang 2016, S. 181). Gleichzeitig wird auch der Gestaltung von „Zwischenräumen“ (Brandt und Bachmann 2014, S. 24), die informelle Kommunikation fördern und damit auch zu einer kommunikativen Atmosphäre an der Hochschule beitragen, mehr Aufmerksamkeit geschenkt werden müssen. In diesen Zwischenräumen können sich Studierende, Lehrende und Mitarbeitende begegnen und es können Situationen entstehen, die sich von dem eher noch hierarchisch strukturierten Lehrenden-Lernenden-Verhältnis unterscheiden. So können auch Raumstrukturen dazu beitragen, die Rahmenbedingungen zu schaffen, aus der Hochschule eine ganzheitliche Lernwelt zu machen. Wie sich die aktuelle Situation an deutschen Hochschulen darstellt, wurde im Forschungsprojekt „Lernwelt Hochschule“ untersucht. 


\section{Lehr- und Lernräume im Kontext der Lernwelt Hochschule}

In dem Maße, in dem die Digitalisierung an den Hochschulen an Bedeutung gewinnt, stellt sich auch die Frage nach der Relevanz physischer Lehr-Lernräume. Nach wie vor gibt es grundlegende Raumnutzungsszenarien, die den Hochschulalltag prägen: Vorlesung, Seminar, Selbstlernaktivitäten der Studierenden in allen Sozialformen, aber auch die Möglichkeit, zu entspannen oder zu kommunizieren, sind solche Nutzungsszenarien. Hinzu kommt, dass sich der studentische Alltag verändert hat. Betrachtet man Megatrends, wie zum Beispiel New Work oder Individualisierung, so wird deutlich, dass es zunehmend zu einer Verschmelzung von Arbeitsprozessen beziehungsweise dem Studium mit sonstigen Aktivitäten kommt. Tradierte Konzepte der Zeitorganisation (fixer Arbeits- oder Veranstaltungsbeginn, Mittagspausen, fixer Endtermin des Arbeits- oder Studientags) verschwinden immer mehr aus der Lebensrealität der Studierenden. Die verschiedenen Aktivitätsbereiche verschwimmen sowohl inhaltlich wie auch organisatorisch. Insbesondere Hochschulen müssen diesen Entwicklungen mit entsprechenden Angeboten begegnen. Dies stellt hohe Anforderungen an die Konzeption von Räumen, die eine wichtige Unterstützungsstruktur für das Lehren und Lernen darstellen - und dies sind eben nicht nur traditionelle Lehr- und Lernräume, sondern auch Orte zur Kommunikation und Entspannung.

Dass sich an den Hochschulen Entwicklungen in Richtung einer ganzheitlichen Perspektive des Blicks auf das Lernen Studierender abzeichnen, hat das Forschungsprojekt „Lernwelt Hochschule“ gezeigt (Becker und Stang 2020a). Hochschulen haben in den vergangenen Jahren Anschaffungen für die flexible Raumnutzung getätigt oder planen diese für die kommenden Jahre (Becker und Stang 2020b). Ebenso wird bei der Konzeption von Selbstlernarealen auf Faktoren wie analoge Unterstützung wie Flipcharts oder Moderationskoffer, digitale Unterstützung mit mobilen Monitoren, Beamern oder interaktiven Multi-User-Screens, Belichtung und Beleuchtung, Vielfalt und Flexibilität der Möblierung, Zonierung in laute und leise Zonen und die Sozialform der Studierenden geachtet, um den situativen Bedürfnissen gerecht zu werden und die Nutzungsmöglichkeiten damit zu erhöhen. Diese Entwicklung geht oft von den Bibliotheken aus, in deren Verantwortung die Gestaltung von Lernflächen für das selbst organisierte Lernen der Studierenden nicht selten fällt. Aschinger stellt jedoch fest, dass ,hochschulweite Aktivitäten sich eher auf die Verfügbarkeit verschiedener Raumarten, weniger auf deren Gestaltung beziehen“ (Aschinger 2020, S. 141).

Es wird deutlich, dass die meisten Hochschulen noch kein ganzheitliches Konzept zur Gestaltung der (potenziellen) Lehr- und Lernflächen entwickelt 
haben. Die SRH Hochschule Heidelberg hat in diesem Bereich eine Vorreiterrolle übernommen und unter Berücksichtigung der Fachkulturen im Rahmen des CORE-Prinzips Lernraumkonzepte für ,,einen ganzheitlichen Handlungsraum entwickelt“ (Ninnemann et al. 2020, S. 188). Ninnemann et al. (2020) machen auch deutlich, dass der Organisation eine bedeutende Rolle zukommt. Für Studierende wird dies vor allem in der Zugänglichkeit der Räume spürbar, die sich in den Öffnungszeiten, den Zugangsberechtigungen, aber auch in der Möglichkeit zeigt, dort zum Beispiel zu essen und zu trinken. Aus der Studierendenperspektive sind eine größtmögliche Flexibilität der Raumszenarien und eine möglichst gute Zugänglichkeit wünschenswert.

Im Forschungsprojekt Lernwelt Hochschule zeigte sich, dass Essen und Trinken in den Zwischenräumen und den eigenständigen Selbstlernzentren unproblematisch gesehen werden. Für die Bibliotheken zeigt sich ein differenzierteres Bild: Dort verbieten $41 \%$ das Essen und Trinken generell und es wurde deutlich, dass oftmals nur das Mitführen von verschließbaren Wasserflaschen, die auch nur Wasser enthalten dürfen, gestattet ist (Becker und Stang 2020b). Selbst in Bibliotheken, die neu gebaut werden, werden abgetrennte Cafés gestaltet, in denen die Studierenden essen und trinken dürfen. Die Studierenden müssen den Lernplatz verlassen, die Bücher oder Medien ausleihen, um dann in dem Café weiterarbeiten zu können, wenn sie einen Kaffee trinken wollen.

Doch nicht nur das Essen und Trinken sind für die Organisation des Lernens von Relevanz, sondern auch die Öffnungszeiten, die zumeist von den allgemeinen Öffnungszeiten der Hochschule abweichen. Denkt man nun an Studierende, die sich aufgrund ihrer Lebensumstände vor Vorlesungsbeginn an der Hochschule aufhalten und die Zeit noch zur Recherche in der Bibliothek nutzen möchten, so entsteht zumindest für die Nutzung des noch nicht digital zugreifbaren Bestands ein Hindernis, das durch einen automatisierten Zugang, zum Beispiel durch den Nachweis der Hochschulzugehörigkeit, gelöst werden könnte.

Ähnlich verhält es sich mit der Zugänglichkeit der Lehrräume, die zum Selbststudium außerhalb der Nutzung für die Lehrveranstaltungen verfügbar sein könnten. Doch oftmals sind Räume verschlossen und bestimmten Nutzendengruppen vorbehalten, da Befürchtungen hinsichtlich der darin befindlichen Technik bestehen. Auch diese Räume könnten, zum Beispiel über ein Raumbuchungssystem, in dem sich Verantwortlichkeit für den Raum verankern lässt, für Individualbeziehungsweise Gruppenlernende geöffnet werden.

Auch wenn Veränderungen in diesen Bereichen schon lange angemahnt werden, zeigt sich, dass diese längst nicht in allen Hochschulen konsequent angegangen werden. Digitalisierung der Lernorganisation könnte hier eine große Hilfe für die Studierenden darstellen, wenn es darum geht, nicht nur das selbst 
organisierte Lernen im digitalen Modus, sondern auch im analogen Modus im physischen Raum zu gestalten. Insgesamt zeigen sich an vielen Hochschulen noch organisatorische Hemmnisse, eine hybride Lernwelt zu gestalten. Die Praxis zeigt, dass es hierzu keinen immensen Aufwand benötigt, sondern die Etablierung eines offeneren Systems. Die Öffnung der physischen Hochschule wird in Bezug auf eine stärkere Studierendenorientierung eine zentrale Stellschraube sein. Doch auch die Frage, wie Räume für innovative Lehr- und Lernformate und veränderte Formen der Betreuung aussehen sollten, wird zu beantworten sein.

\section{Raumdimensionen innovativer Lehr- und Lernformate und Betreuung}

Die Gestaltung innovativer Lehr- und Lernformate und Betreuungskonzepte ist sowohl in physischen als auch in digitalen Kontexten von hoher Relevanz. Der Beantwortung der Frage nach der Gestaltung von hybriden Raumkonzepten wird dabei eine besondere Bedeutung zukommen. Das Digitale soll nicht ohne die physische Raumdimension betrachtet werden, die physische Raumdimension nicht ohne die Möglichkeit, Lehr- und Lernprozesse digital zu unterstützen. Die Verzahnung beider Raumdimensionen zeichnet sich als eine Lösung ab, um den Ad-hoc-Bedürfnissen von Lehrenden und Lernenden im 21. Jahrhundert gerecht $\mathrm{zu}$ werden und ubiquitäres Lernen, eine Verzahnung von formellem und informellem Lernen, zu ermöglichen.

Die Diskussion zu Trendthemen wie E-Learning, Blended Learning, Mobile Learning und Flipped Classroom sollte fortan einer genaueren Betrachtung unterzogen werden. Hierbei geht es um eine differenziertere Beschreibung des Interaktionsverhältnisses zwischen beiden Raumdimensionen und den daraus resultierenden neuen Gestaltungsmöglichkeiten. Die hybride Herangehensweise an Lernobjekte und Raumangebote wird beispielsweise durch den Onlinezugriff auf Informationen ermöglicht,

- um sich von zu Hause aus auf den Besuch am Campus oder in der Bibliothek vorzubereiten und sich dort zurechtzufinden (zum Beispiel Zugriff auf eine Campus-App zur räumlichen Orientierung).

- Im physischen Raum greift der Lernende auf den Onlineraum zurück, um sich wiederum im physischen Raum zurechtzufinden.

- um sich von zu Hause aus in Fachinhalte zu vertiefen (zum Beispiel Zugriff auf Lern-Management-Systeme, elektronische Semesterapparate, E-Books), die entweder in Onlinetests oder in E-Klausuren am Campus abgeprüft werden. 
- Durch den Onlineraum wird der Zugriff auf E-Ressourcen ermöglicht, die entweder online oder in Präsenz einer Wissensüberprüfung unterzogen werden.

- indem vom Campus aus mittels innovativen Lernobjekten (zum Beispiel Augmented-Reality-Brillen, Simulationen) die inhaltliche Einarbeitung in ein Thema in der virtuellen Welt erfahrbar gemacht wird.

- Im physischen Raum rufen Lernende Informationen ab, die online zur Verfügung stehen.

Diese Szenarien wurden beispielhaft ausgewählt, sie stehen für weitere innovative Lehr- und Lernszenarien. Neben den ausgewählten Beispielen zur selbst gesteuerten Interaktion sind auch viele weitere Szenarien denkbar, die zur Interaktion anregen oder/und sich als Gruppenarbeit realisieren lassen (Petschenka 2013).

Um die hybriden Raumdimensionen und einen vielfältigen (Aus-) Gestaltungsraum zu ermöglichen, bedarf es entsprechender Dienstleistungsangebote (Infrastrukturen, Tools, Services) an Hochschulen. Das stetig wachsende digitale Angebot muss in zunehmendem Maße von den zentralen Betriebseinheiten stetig neu bewertet und auf dieser Basis veränderte Angebote entwickelt werden. Die Dienstleistungsangebote sind als Reaktion auf ein verändertes Nutzungsverhalten (mobiles, zeit- und ortsunabhängiges Lernen, Ad-hoc-Bedürfnisse, virale Themen wie Künstliche Intelligenz) zu verstehen. Die Bedürfnisse der Lehrenden und Studierenden spiegeln sowohl die gesellschaftlichen und universitären Veränderungen als auch den technologischen Fortschritt wider.

Betreuungskonzepte unterstützen dabei die Interaktion zwischen den Lernenden, zwischen Lehrenden und Lernenden sowie das selbst gesteuerte, kooperative, formelle und informelle Lernen. Auch hier ist die Verzahnung von physischer und virtueller Raumdimension zu beachten. Folgende Gestaltungsaspekte sind nach Kannenberg und Petschenka (2013) von Relevanz:

Lernaktivitäten: Die Lernaktivitäten, die im Lernraum durchgeführt werden können, sind sowohl virtuell als auch real umsetzbar, ergänzen sich oder sind stark aufeinander bezogen beziehungsweise verweisen auf die jeweils andere Dimension.

Lernressourcen: Lernressourcen sind sowohl im virtuellen als auch im realen Lernraum zugänglich oder komplementär organisiert, grundsätzlich liegen sie für den virtuellen Lernraum in digitaler Form als Dokument zum Download, als Onlinetutorial vor.

Betreuung: Betreuungsaspekte spielen in beiden Fällen eine zentrale Rolle, jedoch ermöglichen virtuelle Komponenten zusätzlich zu den zeitgebundenen 
auch zeit- und ortsungebundene Optionen. Die Betreuung kann daher von einer echten Person oder auch durch einen Avatar ermöglicht werden.

Design: Der virtuelle oder reale Lernraum greift in seiner Gestaltung zentrale Designelemente des jeweils anderen Raums auf (Zugehörigkeit beider Räume zueinander).

Angebote und Dienstleistungen: Lernunterstützende Angebote und Dienstleistungen sollten sowohl virtuelle als auch reale Räume adressieren, zum Beispiel eine reale Sprechstunde und eine elektronische Sprechstunde, um Dienstleistungen nicht einseitig zu verankern.

Inhalte: Sowohl reale als auch virtuelle Angebote und Dienstleistungen müssen in ausreichendem $\mathrm{Ma} ß$ vorhanden sein, ebenso müssen die Inhalte ein bestimmtes Niveau aufweisen (Qualität, Aktualität, Attraktivität), damit das Angebot für die Nutzer*innen interessant ist.

Es ist evident, dass das Thema hybrider Lernraum in einem Kosmos institutioneller, pädagogischer und lerntheoretischer Fragestellungen diskutiert werden muss und als neue Raumdimension Akzeptanz finden sollte. Eine hochschulpolitische und institutionelle Auseinandersetzung mit dem Thema wie auch eine sinnvolle Implementation sind notwendig, um das Thema für die Zukunft in der Lernwelt Hochschule nachhaltig und erfolgreich zu verankern. Dabei gilt es allerdings für Hochschulen auch, die zentralen Stakeholder - die Studierenden - und deren Interessen genauer in den Blick zu nehmen, um ihren Bedürfnissen Rechnung zu tragen.

\section{Student Experience als Grundlage von Planungsprozessen}

Mit dem Shift from Teaching to Learning sollte in Hochschulen der Fokus auf den Lernprozessen und Lernumgebungen der Studierenden liegen. Der Blick auf die damit verbundenen Lernerfahrungen greift allerdings nicht weit genug, um das studentische Erleben an den Hochschulen abzubilden. Der im Angloamerikanischen geprägte Begriff „Student Experience“ geht über die Erfahrungen mit den formalen Lehr- und Lernstrukturen hinaus und umfasst auch Aktivitäten außerhalb der Hochschule. Die ganzheitliche Betrachtung nimmt alle Erfahrungen auf, die Studierende von der Bewerbung, der ersten Begegnung und Ankunft an der Hochschule bis zum Abschluss und den Erfahrungen als Alumni machen:

„Internationally, institutions have adopted the term student experience as a phrase that encompasses not only the academic aspects of teaching, learning and curriculum 
but also student lifestyle and extracurricular activities, academic advice, support and mentoring, and work experiences“ (Benckendorff et al. 2009, S. 85).

Die Faktoren, die die Student Experience ausmachen, differenzieren sich hochschul- und studierendenspezifisch, werden aber auch von übergeordneten und externen Aspekten beeinflusst. Hochschulen unterscheiden sich nach formalen Aspekten wie Größe, Standort, Campus, Gebäudeinfrastrukturen oder Services, um nur einige grundlegende Aspekte zu nennen. Studierende haben sehr individuelle Ausgangssituationen und Rahmenbedingungen, dazu gehören zum Beispiel auch die soziale Einbindung und der Grad der Mobilität je nach Distanz des Wohnorts von der Hochschule. Hochschulübergreifende Entwicklungen wie die Bologna-Reform, die unter anderem die physischen Anwesenheitszeiten in den Hochschulen stark erhöht hat, wirken sich ebenso aus wie externe Faktoren, die durch politische Entscheidungen, wirtschaftlichen Druck oder technologische Entwicklungen bestimmt werden (Benckendorff et al. 2009).

Wie sieht die veränderte Ausgangssituation der Studierenden konkret aus? Sie ist geprägt von einem Nebeneinander unterschiedlicher hochschuldidaktischer Kulturen. Einerseits dominieren vielerorts noch traditionelle von den Lehrenden geprägte Lehr- und Lernszenarien. Es sind aber bereits viele Veränderungen spürbar - Studierende erleben eine Vervielfältigung und Virtualisierung der lernrelevanten Orte und haben ihren Lernradius bereits erweitert. Studierende sind selbstbestimmter, aber auch verantwortlicher in ihren Lernprozessen. Ob im Seminarraum, im Gruppenraum der Bibliothek, dem Hochschulcafé oder zu Hause - Flexibilität und Mobilität sind für die Studierenden zunehmend wichtig. Die Grenzen zwischen formalem und informellem Lernen sind fließend. Verlässliche und komfortable digitale Infrastrukturen und Services bilden auch im physischen Raum für die Studierenden die Basis ihrer Lernaktivitäten.

Im angloamerikanischen Raum, in Skandinavien und den Niederlanden gibt es bereits weitreichende Erfahrungen in der systematischen Gestaltung von Hochschulen zu Lernräumen (Weichert 2020); erfolgreiche Konzeptelemente (Gruppenräume, Differenzierung, Zonierung et cetera) wurden identifiziert. Die Erfahrungen haben gezeigt, dass eine bloße Übernahme der Konzeptelemente nicht ausreicht: „I suspect that much insightful knowledge about learning spaces in higher education will not arrive packaged in that way" (Elkington und Bligh 2019, S. 6).

Lernraumkonzepte müssen differenziert unter Beteiligung der Stakeholder aus den Bereichen der Lehre, der Verwaltung, der Einrichtungen wie Bibliothek, Rechenzentrum oder Studierendenservice entwickelt werden (UCISA 2016, S. 16), nur so gelingt der ganzheitliche und spezifische Blick auf die Hochschule. 
Um die Lehr- und Lernprozesse aus der Perspektive der Lernenden zu entwickeln, bedarf es an Hochschulen eines neuen Paradigmas - der konsequenten Studierendenorientierung bei der Gestaltung von Angeboten und Raumszenarien. Studierende werden in diesem Kontext entsprechend zu Hauptakteur*innen - Key Stakeholder. Die Auseinandersetzung mit der Student Experience erlaubt die Annäherung an die studentische Perspektive. Neben der Fachkultur, der Informations- und Lernkultur ist besonders die emotionale und soziale Ebene des Lernens für die Studierenden wichtig, Studierende haben ein großes Bedürfnis nach einer „Heimat“ im akademischen Kontext (Gläser und Kobsch 2020). Dafür ist unabdingbar, die Situation, Wünsche und Bedürfnisse der Studierenden zu kennen. Zur Erforschung der Student Experience ist ein Mix aus quantitativen und qualitativen Verfahren sinnvoll. Quantitative Studien bieten eine gute Basis, um messbare, repräsentative Aussagen zu erhalten. Qualitative Methoden bieten mehr Offenheit und die Möglichkeit, die Studierenden partizipativ einzubinden. Fokusgruppen, Workshops, Interviews und Beobachtungen sind hierfür erprobte Formate (Foster 2007).

Partizipation kann auch durch strukturelle Elemente erreicht werden, dies bezieht sich auf Funktionen, Gremien und die regelhafte Einbindung in Prozesse. Hier spielt auch das bewusste Management des Student-Life-Cycles eine Rolle. Studierende sind bereits in Erhebungen zur Qualität der Lehre und Akkreditierungsprozesse eingebunden und an Gremien beteiligt. Es gibt an einigen Hochschulen sogar das Amt der/des studentischen Vizepräsident*in, die einen entscheidenden und deutlich sichtbaren Schritt zur Studierendenorientierung dokumentieren. Alle Studierendenservices sowie Raumsettings müssen sich daran messen lassen, welche Auswirkungen sie auf die Lernsituation der Studierenden haben. Hierfür sollten die Hochschulen wirksame Strukturen und Prozesse schaffen.

Die Ergebnisse des Projekts Lernwelt Hochschule zeigen, dass die Studierenden bisher nicht systematisch von den Hochschulen in die Gestaltungsprozesse eingebunden werden (Becker und Stang 2020b). Hier besteht für die Hochschulen ein großes Potenzial, um künftige Lernraumentwicklungen wirklich ganzheitlich zu gestalten.

\section{$6 \quad$ Perspektiven für hybride Lehr- und Lernraumkonstellationen}

Insbesondere durch den Einzug digitaler Technologien haben sich in den letzten Jahren Änderungen im Nutzungsverhalten ergeben, welche sich auch auf das 
Lehren und Lernen an Hochschulen auswirken. Vor allem durch die Auswirkungen der Coronapandemie hat sich die Entwicklung dynamisiert. Die Einbeziehung dieser Technologien kann zum einen in den universitären Veranstaltungen in einem formellen Lehr- und Lernszenario erfolgen oder auch in einem informellen Bereich des Lehrens und Lernens, welcher ein entscheidender Faktor im digitalen Zeitalter darstellt. Die wachsende Bedeutung informeller Lernprozesse zeigt, dass formelles und informelles Lernen ebenso wie Präsenz- und Fernlehre, klassische Lernmedien und technologiegestütztes Lernen in intelligenten neuen Modellen zu integrieren sind. Es gilt, diese Modelle für das Lernen und für das wissenschaftliche Arbeiten fruchtbar zu machen.

Die Relevanz physischer und digitaler Raumszenarien wird vor allem angesichts der Entwicklung hybrider Lehr- und Lernraumkonstellationen zunehmen. Die Integration der verschiedenen Ebenen unter Ausnutzung ihrer jeweiligen Potenziale ist allerdings kein triviales Unterfangen (Günther et al. 2019). Vielmehr wird die konzeptionelle Arbeit daran die Hochschulen insgesamt herausfordern. Onlinelehre und -lernen werden keine Alternativen zu Lehr- und Lernkontexten im Präsenzmodus darstellen. Außerdem werden Serviceleistungen wie Literaturbeschaffung, Zugang zu Datenbanken, Beratungen zur Gestaltung des individuellen Lernportfolios ebenfalls in hybride Strukturen überführt werden müssen. Hochschulbibliotheken haben längst damit begonnen, andere Servicebereiche der Hochschule haben hier noch Nachholbedarf.

Bei der Organisation Hochschule wird es darum gehen, die verschiedenen Logiken und Kulturen von Verwaltung, Forschung, Lehre, IT-Abteilungen, Infrastrukturabteilungen et cetera so aufeinander abzustimmen, dass die Lernwelt Hochschule ganzheitlich gestaltet wird. Dabei wird vor allem eine einheitliche Strategie für das Ineinandergreifen physischer und digitaler Aspekte der Hochschulentwicklung zu gestalten sein, um grundlegende Orientierungen zu liefern. Die hektische Betriebsamkeit bei der Vorbereitung eines digitalen Sommersemesters 2020 hat gezeigt, dass es übergreifend an solchen Strategien fehlt. Die Fokussierung auf technische Lösungen ließ offensichtlich werden, dass es an Konzepten der Integration digitaler und physischer Lehr-Lernsettings auch unter einer didaktischen Perspektive fehlt.

\section{Verschränkung von physischen und digitalen Lernräumen als strategisches Konzept}

Obwohl der Fokus der Hochschulen bislang auf dem physischen Lehr-Lernraum liegt, ist evident, dass die Diskussion über virtuelle Lernräume insbesondere 
im digitalen Zeitalter von Bedeutung ist. Die vielerorts realisierten innovativen Konzepte legen eine Erweiterung des hybriden Gedankens hinsichtlich einer sinnvollen Verschränkung von physischen und digitalen Raumdimensionen nahe. Eine solche Interaktion des physischen und des digitalen Lernraums muss jedoch als gesamtstrategisches Konzept erkannt und von der Hochschulleitung forciert werden. Die Coronapandemie ist zugleich Treiberin und Innovatorin für eine neue Ära einer hybriden Raumkultur, die nunmehr auf die Probe gestellt wird.

Im Rahmen des Projektes Lernwelt Hochschule wurden bereits vor der Dynamisierung dieser Entwicklung die Herausforderungen einer zukunftsorientierten Gestaltung von Hochschulen benannt (Stang et al. 2020). Dabei zeigt sich, dass genau die Aspekte als Probleme herausgearbeitet wurden, die sich auch in der Coronapandemie als Probleme herausgestellt haben. Der im Rahmen des Projektes erstellte Baukasten für Veränderungsprozesse an Hochschulen (Petschenka et al. 2020) liefert wichtige Hinweise für die Entwicklung einer ganzheitlichen Strategie für Hochschulen, um die Lernwelt Hochschule und damit auch hybride Lehr- und Lernraumkonstellationen so zu gestalten, dass schnell auf Herausforderungen - welcher Art auch immer - reagiert werden kann.

\section{Literatur}

Aschinger, F. (2020). Konzeption und Management der Lernwelt Hochschule. Herausforderungen und Good Practice aus Sicht der Hochschulakteurinnen und -akteure. In A. Becker \& R. Stang (Hrsg.), Lernwelt Hochschule. Dimensionen eines Bildungsbereichs im Umbruch (S. 123-149). Berlin: De Gruyter Saur.

Aschinger, F., Becker, A., Gageur, N., \& Weichert, H. (2020). Forschungsfeld Lernwelt Hochschule. Methodische Zugänge zur Analyse einer differenzierten Struktur. In A. Becker \& R. Stang (Hrsg.), Lernwelt Hochschule. Dimensionen eines Bildungsbereichs im Umbruch (S. 19-46). Berlin: De Gruyter Saur.

Becker, A., \& Stang, R. (Hrsg.). (2020). Lernwelt Hochschule. Dimensionen eines Bildungsbereichs im Umbruch. Berlin: De Gruyter Saur.

Becker, A., \& Stang, R. (2020). Lernwelt Hochschule im Aufbruch. Zentrale Ergebnisse einer Befragung. In A. Becker \& R. Stang (Hrsg.), Lernwelt Hochschule. Dimensionen eines Bildungsbereichs im Umbruch (S. 71-125). Berlin: De Gruyter Saur.

Benckendorff, P., Ruhanen, L., \& Scott, N. (2009). Deconstructing the student experience. A conceptual framework. Journal of Hospitality and Tourism Management, 16, 84-93. https://doi.org/10.1375/jhtm.16.1.84.

Brandt, S., \& Bachmann, G. (2014). Auf dem Weg zum Campus von morgen. In K. Rummler (Hrsg.), Lernräume gestalten. Bildungskontexte vielfältig denken (S. 15-28). Münster: Waxmann.

Elkington, S., \& Bligh, B. (2019): Future learning spaces: Space, technology and pedagogy. Heslington: AdvanceHE. https://s3.eu-west-2.amazonaws.com/assets.creode.advancehe- 
document-manager/documents/hea/private/downloads/Future\%20Learning\%20Spaces_ 1_1568037687.pdf. Zugegriffen: 19. Okt. 2020.

Foster, N. F, \& Gibbons, S. (2007). Studying students. The undergraduate research project at the University of Rochester. Chicago: ACRL. https://www.ala.org/acrl/sites/ala.org. acrl/files/content/publications/booksanddigitalresources/digital/Foster-Gibbons_cmpd. pdf. Zugegriffen: 19. Okt. 2020.

Gläser, C., \& Kobsch, L. (2020). Student Experience in der Lernwelt Hochschule. Studierende im Fokus der Fallstudien. In A. Becker \& R. Stang (Hrsg.), Lernwelt Hochschule. Dimensionen eines Bildungsbereichs im Umbruch (S. 150-169). Berlin: De Gruyter Saur.

Günther, D., Kirschbaum, M, Kruse, R., Ladwig, T., Prill, A., Stang, R., \& Wertz, I. (2019). Zukunftsfähige Lernraumgestaltung im digitalen Zeitalter. Thesen und Empfehlungen der Ad-hoc Arbeitsgruppe Lernarchitekturen des Hochschulforum Digitalisierung (Arbeitspapier, 44). Berlin: Hochschulforum Digitalisierung. https://doi.org/10.5281/zenodo.348 4678.

Jelich, F.-J., \& Kemnitz, H. (Hrsg.). (2003). Die pädagogische Gestaltung des Raums. Geschichte und Modernität. Bad Heilbrunn: Klinkhardt.

JISC. (2006). Designing spaces for effective learning. A guide to 21st century learning space design. Bristol: JISC. https://www.webarchive.org.uk/wayback/archive/201406 13220103/http://www.jisc.ac.uk/media/documents/publications/learningspaces.pdf.

Zugegriffen: 19. Okt. 2020.

Kannenberg, S., \& Petschenka, A. (2013). Virtuelle Lernräume. In Deutsche Initiative für Netzwerkinformation e. V. (Hrsg.), Die Hochschule zum Lernraum entwickeln. Empfehlungen der DINI AG Lernräume (S. 69-84). Kassel: University Press.

Kraus, K., Stang, R., Schreiber-Barsch, S., \& Bernhard, C. (2015). Erwachsenenbildung und Raum. Eine Einleitung. In C. Bernhard, K. Kraus, S. Schreiber-Barsch, \& R. Stang (Hrsg.), Erwachsenenbildung und Raum. Theoretische Perspektiven - Professionelles Handeln - Rahmungen des Lernens (S. 11-25). Bielefeld: W. Bertelsmann.

Löw, M. (2012). Raumsoziologie (7. Aufl.). Frankfurt a. M.: Suhrkamp.

Ninnemann, K., Rózsa, J., \& Sutter, C. (2020). Zur Relevanz der Verknüpfung von Lernen, Raum und Organisation. In R. Stang \& A. Becker (Hrsg.), Zukunft Lernwelt Hochschule. Perspektiven und Optionen für eine Neuausrichtung (S. 176-187). Berlin: De Gruyter Saur.

Nugel, M. (2015). Sich entwerfen in, mit und durch Raum. Bildungstheoretische Überlegungen zur Architektur der Erwachsenenbildung. In C. Bernhard, K. Kraus, S. Schreiber-Barsch, \& R. Stang (Hrsg.), Erwachsenenbildung und Raum. Theoretische Perspektiven - Professionelles Handeln - Rahmungen des Lernens (S. 55-65). Bielefeld: W. Bertelsmann.

Petschenka, A. (2013). Virtuelle Lernräume als Aufgabe von Hochschulbibliotheken. Entwicklungen, Praxis, Perspektiven, (Masterarbeit: TH Köln). https://publiscologne.thkoeln.de/frontdoor/deliver/index/docId/124/file/MAT_Petschenka_Anke.pdf. Zugegriffen: 19. Okt. 2020.

Petschenka, A., Stang, R., Becker, A., Franke, F., Gläser, C., Weckmann, H.-D., \& Zulauf, B. (2020). Die Zukunft der Lernwelt Hochschule gestalten. Ein Baukasten für Veränderungsprozesse. In R. Stang \& A. Becker (Hrsg.), Zukunft Lernwelt Hochschule. Perspektiven und Optionen für eine Neuausrichtung (S. 213-256). Berlin: De Gruyter Saur. 
Stang, R. (2016). Lernwelten im Wandel. Entwicklungen und Anforderungen bei der Gestaltung zukünftiger Lernumgebungen. Berlin: De Gruyter Saur.

Stang, R. (2017). Analoger Körper im digitalen Raum. Lernen im Zeichen einer ambivalenten Kontextualisierung. In F. Thissen (Hrsg.), Lernen in virtuellen Räumen. Perspektiven des mobilen Lernens (S. 28-38). Berlin: De Gruyter Saur.

Stang, R., \& Becker, A. (Hrsg.). (2020). Zukunft Lernwelt Hochschule. Perspektiven und Optionen für eine Neuausrichtung. Berlin: De Gruyter Saur.

Stang, R., Becker, A., Franke, F., Gläser, C., Petschenka, A., Weckmann, H.-D., \& Zulauf, B. (2020). Herausforderung Lernwelt Hochschule. Perspektiven für eine zukünftige Gestaltung. In A. Becker \& R. Stang (Hrsg.), Lernwelt Hochschule. Dimensionen eines Bildungsbereichs im Umbruch (S. 182-210). Berlin: De Gruyter Saur.

UCISA - Universities and Colleges Information System. (2016). The UK higher education learning space toolkit. https://www.ucisa.ac.uk/-/media/337970AB91674EA38B5C267F 062A10F5.ashx. Zugegriffen: 19. Okt. 2020.

Weichert, H., \& Stang, R. (2020). Der Blick von außen. Einschätzungen internationaler Expertinnen und Experten. In A. Becker \& R. Stang (Hrsg.), Lernwelt Hochschule. Dimensionen eines Bildungsbereichs im Umbruch (S. 170-180). Berlin: De Gruyter Saur.

Open Access Dieses Kapitel wird unter der Creative Commons Namensnennung 4.0 International Lizenz (http://creativecommons.org/licenses/by/4.0/deed.de) veröffentlicht, welche die Nutzung, Vervielfältigung, Bearbeitung, Verbreitung und Wiedergabe in jeglichem Medium und Format erlaubt, sofern Sie den/die ursprünglichen Autor(en) und die Quelle ordnungsgemäß nennen, einen Link zur Creative Commons Lizenz beifügen und angeben, ob Änderungen vorgenommen wurden.

Die in diesem Kapitel enthaltenen Bilder und sonstiges Drittmaterial unterliegen ebenfalls der genannten Creative Commons Lizenz, sofern sich aus der Abbildungslegende nichts anderes ergibt. Sofern das betreffende Material nicht unter der genannten Creative Commons Lizenz steht und die betreffende Handlung nicht nach gesetzlichen Vorschriften erlaubt ist, ist für die oben aufgeführten Weiterverwendungen des Materials die Einwilligung des jeweiligen Rechteinhabers einzuholen.

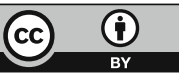

